\title{
Facial Recognition understanding and Differences Between PCA (Principal Component Analysis) and LDA (Linear Discriminant Analysis)
}

\author{
Aarav Yadav ${ }^{1}$, Ms. Anamika Jain ${ }^{2}$ \\ UG Student, Computer Science Department, Poornima Group of Institutions, Jaipur, Rajasthan, India ${ }^{1}$ \\ Asst. Professor, Computer Science Department, Poornima Group of Institutions, Jaipur, Rajasthan, India ${ }^{2}$
}

\begin{abstract}
Facial recognition is the process of identification of humans by the beyond wildest dreams characteristics of their Faces. Face recognition technology is the end intrusive and fastest bio-metric technology to identify the human face. With increasing money in the bank needs and by all of advancement in technology extracting taste has become roughly simpler. Authentication by passwords are not secure, so this technology are much more important as for upcoming security and safety measures. Many technologies have been developed for finding the best possible ways to describe the face descriptors and finding the face from available database of images. In this Paper we have discussed some understanding about Facial Recognition and differences between two algorithms which are used for facial description, these are PCA (Principal Component Analysis) and LDA (Linear Discriminant Analysis).
\end{abstract}

Keywords: Face Recognition; PCA Algorithm; LDA Algorithm; Image Processing.

\section{INTRODUCTION}

Automatic face analysis which includes, e.g., find based, which uses holistic texture features and is applied to detection, face recognition, and facial allusion recognition either whole-face or specific face image and feature-based, has acquired an as a matter of fact active tenor in computer which uses geometric facial features (mouth, eyebrows, vision research. A key deliver in face analysis is finding cheeks etc..), and geometric relationships between them. If feasible descriptors for face appearance.The human brain a face is well-known it is experienced or the program may has numerous fully interconnected biological neurons show a similar face existing in database else it is unknown. which, on several specific tasks, boot outperform super In software if unknown face is there and software does not computers. A child can accurately view a face, anyhow for matches any face then it is stacked in database for further a personal digital assistant it is a troublesome task. Early recognition. These steps are very useful in criminal face recognition algorithms hand me down easily done identification. In general, face recognition uses alternative geometric models, but in a different way the recognition texture features and is applied to as a choice whole-face or behavior has rapidly matured into an information of specific face image and feature-based, which uses imposing mathematical representations and related geometric facial features (mouth, eyebrows, cheeks etc.), processes. Major advancements and initiatives have and geometric relationships surrounded by them.

propelled contact recognition technology into the Principal Component analysis (PCA) is such of the most spotlight.Computers that recognize and recognize faces popular holistic approach i.e. appearance-based methods could be try a wide fluctuation of practical applications used for dimensional reduction for compression and face including felon identification etc. Face detection and recognition problems. Linear discriminant analysis (LDA) recognition is used in multiple places nowadays,verifying is another strapping dimensional reduction technique which websites hosting images and urban networking sites. is further known as fisher's discriminant analysis. It has Features extracted from a face are processed and compared been used extensively in multiple applications one as face by all of similarly qualified faces reveal in the database. If recognition, image retrieval etc.The PCA method was a face is recognized then the system may show a face developed in 1991 [7]. The PCA method is used for existing in database else it is unknown. In surveillance dimension reduction for linear discriminate analysis system if an unknown face appears more than one time (LDA), generating a new paradigm, and called fisherface. then it is stored in database for further recognition. The The fisherface approach is more insensitive to variations of steps are used in criminal face identification. In general, lighting, illumination and facial expressions. However, this face recognition techniques can be divided into two groups approach is more computationally expensive than the PCA based on the face representation they use appearanceapproach. 
In this paper there are several sections where in section II, localized and normalization process may be sanctioned to some idea of the Face Recognition System. In section III, draw the dimensions of the live facial sample in alignment feature extraction technique by Principal Component by the whole of the one on the template. After the face is Analysis and LDA Linear Description Analysis. In section detected then it is compared with the database image and IV, Comparison is done between the LDA and PCA. In with feature extraction result it results the known or section V, Conclusion and Future research directions are unknown image class. discussed.

\section{FACE RECOGNITION SYSTEM}

Face recognition system consists of four steps, as shown in Fig.1 Image acquisition, pre-processing, feature extraction and classification as known or unknown [4].

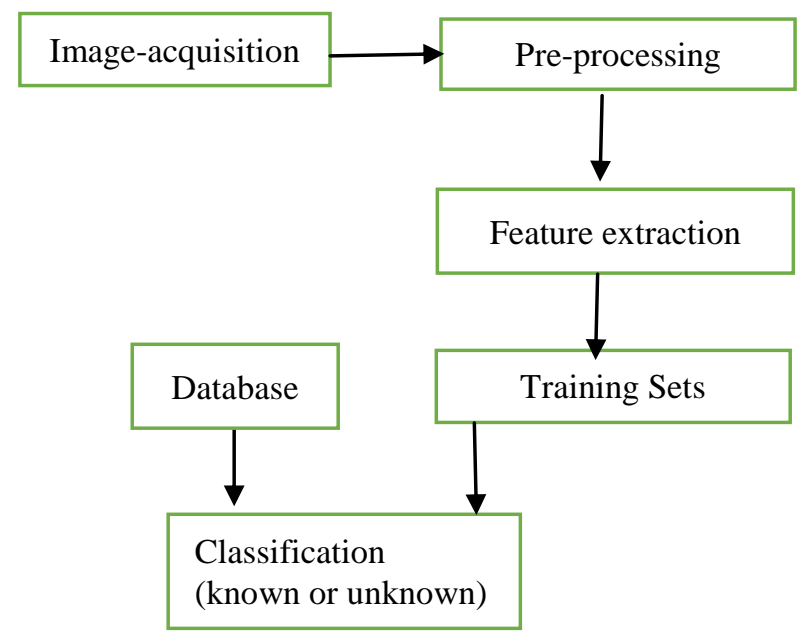

Figure 1. Face Recognition System

Many methods have been developed and presented to improve face recognition systems. K.C.Lee et al.[1] presented a paper that shows how to arrange physical lighting so that the acquired images of each object can be directly used as the basis vectors of a low-dimensional linear space and that this subspace is close to those acquired by the other methods.Margarita Osadchy et al.[2] show that the surface characteristics determine the type of image comparison method that should be used. Jian Yang et al.[6] presented a new technique, coined twodimensional principal component analysis (2DPCA) based on $2 \mathrm{D}$ image matrices rather than $1 \mathrm{D}$ vectors so the image matrix does not need to be transformed into a vector prior to feature extraction.

For face recognition matter of choice is verification. This is where the program compares the given individual with who that individual says they are and gives a yes or no decision. The input can be recorded audio tape of the speaker or a besides image. A sample of $1 \mathrm{sec}$ length consists of a 25 figure video sequence. More than one camera can be used to serve a $3 \mathrm{D}$ cross section of the face and to preserve against the usage of photographs to gain unauthorized access.A pre-processing module locates the eye position and takes service of the surrounding lighting element and color variance. First the presence of faces or face in a scene intend be detected. Once the face is detected, it must be

\section{EXTRACTION TECHNIQUES USING PCA AND LDA}

In this section two techniques have been described with the algorithm that how they works. They both are dimensionality reduction technique used for facial recognition.

\section{A. PCA- Principal Component Analysis}

Principal component analysis (PCA) is a dimensionality reduction technique. It is also known as eigenspace projection or karhunen-loeve transformation [7].PCA calculates the Eigen vectors of the covariance matrix, and projects the natural data onto lessen dimensional feature space, which is bounded by Eigen vectors mutually large Eigen values. PCA has been used in face representation and description where the Eigen vectors calculated are named as Eigen faces.It is a well- known of the more profitable techniques of face recognition.PCA is a satisfying statistical course that has found application in fields one as face recognition and image compression, and is a mean technique for inquiry patterns in disclosure of high dimension..

The profit of PCA is reduce dimensionality of the data. No data redundancy is found as components are orthogonal. With help of PCA, complexity of grouping the images can be reduced.

Steps for this technique: -[5][7]

Step 1: Create a training set. Training set consist of total $\mathrm{M}$ images and each image is of $\mathrm{N}^{*} \mathrm{~N}$.

Step 2: Convert the face images in the training set to face vector denoted as $\mathrm{Xi}$.

Step 3: Normalize the face vector. First calculate the average face vector and then subtract average face vector from each face vector.

Normalized face vector $\Phi \mathrm{i}=\mathrm{Xi}-\psi$

Step 4: now find Eigen faces with help of covariance matrix.

$\mathrm{C}=\mathrm{AA}^{\mathrm{t}}$ Where $\mathrm{A}=\{\Phi 1, \Phi 2 \ldots \Phi \mathrm{m}\}$

Step 5: Calculate eigenvectors from a above matrix with reduced dimensionality.

Step 6: Select $\mathrm{K}$ best eigenfaces, as such $\mathrm{K}<M$ and can represent the whole training set.

Step 7:Convert lower dimensional $\mathrm{K}$ eigenvectors into original face dimensionality $\mathrm{U}_{\mathrm{i}}=\mathrm{AV}_{\mathrm{i}}$

Here $U_{i}=i$ th vector in higher dimension space and $V_{i}=i$ th vector in lower dimension space

Step 8: Represent the each face image a linear combination of all $\mathrm{K}$ eigen vectors and each face from the image can be represented as a weighted sum of $\mathrm{K}$ eigenfaces + mean or average face. 
Step 9: Now recognition using PCA.

\section{B. LDA- Linear Discriminant Analysis}

The end of Linear discriminant experiment is to classify divide labels by the method of group, customers, machinery, and whole other into such or two or greater groups based on a set of features that explain the objects e.g. gender, decrepitude, profit, weight, preference conclude, etc. If one can assume that the groups are linearly separable, one can consider linear discriminant model (LDA).Linearly separable suggests that the groups can be sole by a linear combination of features that represent the objects.

If solo two features, the separators between objects accumulation will acquire lines. If the number of features is three, the separator is a plane and if the number of features i.e. individualistic variables is more than three, the separators acquire a hyper-plane.

Linear discriminant experiment (LDA) is generally used course for image classification and dimensionality reduction. Linear discriminant experiment is further known as fisher's discriminant experiment and it searches for those vectors in the inherent space that best discriminate in classes. The intention of LDA is to pound dimensionality reduction while preserving as a round of the class discriminatory idea as possible. The function of LDA is to maximize the between-class scatter matrix measure at the same time minimizing the within-class scatter matrix measure [3].

There are few steps which are followed to perform the LDA technique:-

Step 1: Compute the d-dimensional mean vectors for the different classes from the dataset.

Step 2: Compute the scatter matrices (between-class and within-class scatter matrix).

Step 3: Compute the eigenvectors (e1, e2... ed) and corresponding eigenvalues $(\lambda 1, \lambda 2 \ldots \lambda d)$ for the scatter matrices

Step 4: Sort the eigenvectors by decreasing eigenvalues and choose $\mathrm{k}$ eigenvectors with the largest eigenvalues to form a dxk-dimensional matrix $\mathrm{W}$ (where every column represents an eigenvector).

Step 5: Use this $\mathrm{d} \times \mathrm{k}$ eigenvector matrix to transform the samples onto the new subspace.

This can be summarized by the mathematical equation: $\mathrm{y}=\mathrm{WT} \times \mathrm{x}$ (where $\mathrm{x}$ is a $\mathrm{d} \times 1$-dimensional vector representing one sample, and $\mathrm{y}$ is the transformed $\mathrm{k} \times 1$ dimensional sample in the new subspace). [8]

Step 9: Now recognition is done using LDA.

\section{DIFFERENCES BETWEEN PCA AND LDA}

1. PCA is slight sensitive whereas LDA is preferably sensitive.PCA takes absolutely less computational time whereas LDA takes greater computational time.

2. LDA is based on a single face image as input. That manner LDA can perform face description for a single input image; hereafter it does not concern multiple input images. Whereas PCA is based on countless face images as input. Hence it considers endless input images.

3. In LDA algorithm we have to calculate two scatter matrix which are within-class and between-class scatter matrix.

4. But in PCA we only need one scatter matrix to calculate Eigen value. Therefore LDA algorithm needs much more time than PCA.

5. We can also say that LDA is supervised learning whereas PCA is unsupervised learning as in PCA algorithm while extracting features from face and matching it ignores class labels and LDA keeps the class label in consideration.

6. PCA is much more suitable than LDA when we have small number of samples per class but when the number of samples increases then LDA might over perform the PCA (as LDA directly deals with class discrimination).

\section{CONCLUSION AND FUTURE SCOPE}

In this paper, a survey on the PCA and LDA and comparison of both onwhat it is all about of their merits and demerits. We analyses that at which point PCA and LDA work on images and compared both techniques and found that PCA is better as compared to LDA.

Both techniques are used for the dimensional reduction and both are remarkable in applications like security, investigation etc. So as compared here we can say that combination of both the algorithms PCA as well as LDA will get the best and effective results, so without preferring any other algorithm to determine face combination should be taken in consideration. Moreover if new algorithm has to be made then demerits of PCA and LDA has to be taken care of.

\section{REFERENCES}

[1] K.C.Lee, J.Ho and D.J. Kriegman( 2005) "Acquiring Linear Subspaces for Face Recognition under Variable Lighting" IEEE TRANSACTIONS ON PATTERN ANALYSIS AND MACHINE INTELLIGENCE, VOL. 27, NO. 5, MAY 2005

[2] Margarita Osadchy, David W. Jacobs and Michael Lindenbaum (2007) "Surface Dependent Representations for Illumination Insensitive Image Comparison" Ieee Transactions On Pattern Analysis And Machine Intelligence, VOL. 29, NO. 1, JANUARY 2007

[3] Martinez A.M. and Kak A.C., "PCA versus LDA", IEEE Transactions on Pattern Analysis and Machine Intelligence, Vol. 23, No.2, pp. 228-233, 2001.

[4] M.Sathish Kumar, S.Sangeetha(2014) "Blur and Illumination robust face recognition using Bayes classifier" International Journal Of Innovative Research In Electrical, Electronics, Instrumentation And Control Engineering Vol. 2, Issue 1, January 2014.

[5] Sezin Kaymak "Enhanced Principal Component Analysis Recognition Performance"

[6] Jian Yang, Zhang. D, Frangi, A.F. and Jing-Yu Yang (2004) "Twodimensional PCA: a new approach to appearance-based face representation and recognition". Pattern Analysis and Machine Intelligence, IEEE Transactions on (Volume:26, Issue: 1) Page(s): 131 - 137

[7] M.Turk and A.Pentland, "Eigenfaces for Recognition", Journal of Congnitive Neuroscience, 3(1), pp.71-86, 1991. 
[8] Changjun Zhou*, Lan Wang, Qiang Zhang, Xiaopeng Wei "Face recognition based on PCA image reconstruction and LDA" Optik 124 (2013) 5599- 5603.

[9] S. Thakur,J. K. Sing,D. K. Basu, M. Nasipuri,M. Kundu”Face Recognition using Principal Component Analysis and RBF Neural Networks",IJSSST, Vol. 10, No. 5.

[10] Suman Kumar Bhattacharyya,Kumar Rahul " Face Recognition By Linear Discriminant Analysis", International Journal of Communication Network Security, ISSN: 2231 - 1882, Volume-2, Issue-2, 2013.

[11] Tiwari Priti Anilkumar, Kalyani Jha, Karishma P Uchil, Naveen H,'Haar Features Based Face Detection and Recognition for Advanced Classroom and Corporate Attendance",International Journal of Innovative Research in Computer and Communication Engineering,Vol. 3, Issue 5, May 2015.

[12] Timo Ahonen,Matti Pietikainen, "Face Description with Local Binary Patterns: Application to Face Recognition", Ieee Transactions On Pattern Analysis And Machine Intelligence, Vol. 28, No. 12, December 2006. 